\title{
Propyl isothiocyanate induces apoptosis in gastric cancer cells by oxidative stress via glutathione depletion
}

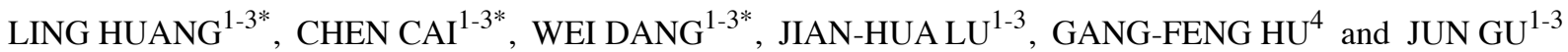 \\ ${ }^{1}$ Department of General Surgery, Xinhua Hospital Affiliated to Shanghai JiaoTong University School of Medicine; \\ ${ }^{2}$ Shanghai Key Laboratory of Biliary Tract Disease Research; ${ }^{3}$ Shanghai Research Center of Biliary Tract Disease, \\ Shanghai 200092; ${ }^{4}$ Department of General Surgery, Chongming Branch, Xinhua Hospital Affiliated to \\ Shanghai JiaoTong University School of Medicine, Shanghai 202150, P.R. China
}

Received February 17, 2019; Accepted August 20, 2019

DOI: $10.3892 / \mathrm{ol} .2019 .10875$

\begin{abstract}
Isothiocyanates are a group of compounds that exist in the majority of cruciferous plants. A number of isothiocyanates have been demonstrated to exhibit anticancer effects; however, antitumor properties of propyl isothiocyanate (PITC) have not been evaluated previously. In this study, the possible effects of PITC on gastric cancer (GC) cells were investigated, and the potential underlying mechanisms were explored. The results demonstrated that PITC inhibited cell viability of two GC cell lines and induced cell cycle arrest and apoptosis. Treatment with PITC promoted total glutathione depletion in GC cell lines, leading to reactive oxygen species accumulation and DNA damage, which activated the mitochondria-dependent and p53 signaling pathways to trigger apoptosis in GC cells. The effects of PITC were reversed by N-Acetyl-L-cysteine. The results of the present study revealed the potential mechanisms of PITC on apoptosis induction in GC cells, which may be mediated by mitochondria-dependent apoptosis and DNA damage.
\end{abstract}

\section{Introduction}

Gastric cancer (GC) is the second most common cause of cancer-associated mortality and the second most common

Correspondence to: Professor Jun Gu, Department of General Surgery, Xinhua Hospital Affiliated to Shanghai JiaoTong University School of Medicine, 1665 Kongjiang Road, Shanghai 200092, P.R. China

E-mail: gujun02@xinhuamed.com.cn

Mr. Gang-Feng Hu, Department of General Surgery, Chongming Branch, Xinhua Hospital Affiliated to Shanghai JiaoTong University School of Medicine, 25 Nanmen Road, Chengqiao, Chongming, Shanghai 202150, P.R. China

E-mail: eric1943@vip.qq.com

${ }^{*}$ Contributed equally

Key words: propyl isothiocyanate, human gastric cancer, apoptosis, reactive oxygen species, mitochondria-dependent pathway type of malignancy in China (1). Although the nationwide incidence and mortality rates of gastric cancer in China have decreased in recent years, with an incidence rate of $10.79 \%$ and a mortality rate of $12.8 \%$ in 2014 (2), it remains a major public health problem. Due to the high rate of recurrence or metastasis, the 5-year survival rate of GC is only 5-20\% (3). Chemotherapy before or after a surgical resection is a crucial adjuvant treatment for advanced GC, providing a method to expand patients' lifetime (4). In the last several decades, new methods have been sought to improve the prognosis of advanced GC, whereas the standard interventions have not been changed. Therefore, novel pharmaceuticals are urgently needed to improve the outcomes of patients with advanced GC.

Propyl isothiocyanate (PITC) is one of the natural isothiocyanates (ITCs) with the characteristic chemical group $-\mathrm{N}=\mathrm{C}=\mathrm{S}$ (Fig. 1A). Natural ITCs are generally the hydrolysates of glucosinolates (GLS), which are innocuous and widely exist in cruciferous plants (5). While GLS are non-toxic, ITCs exert toxicity by interacting with thiol- and amine-groups in peptides and proteins (6), exhibiting a wide spectrum of bioactivities, including anticancer properties (7). Glutathione (GSH) and several mitochondrial proteins, such as ATP synthase (Complex I), cytochrome c oxidase (Complex IV) and thioredoxin-dependent peroxide reductase have been identified as potential ITC targets, the inhibition of which may lead to reactive oxygen species (ROS) accumulation (8-10). Certain ITCs, such as sulforaphane, allyl ITC (AITC), benzyl ITC and phenethyl ITC have been demonstrated to effectively inhibit cell proliferation and induce apoptosis of multiple types of cancer cells (11-14). However, no detailed studies have investigated the bioactivity of PITC in vitro and in vivo. In the present study, the anti-neoplastic activity of PITC in GC cell lines (MGC-803 and HGC-27) was investigated to provide insight into the molecular mechanisms involved, which may be the experimental evidence for using PITC as a new natural anti-cancer medicine for GC.

\section{Materials and methods}

Drugs and antibodies. PITC and dimethyl sulfoxide (DMSO) were purchased from Sigma-Aldrich; Merck KGaA. 
$\mathrm{N}$-acetyl-L-cysteine (NAC) was purchased from Beyotime Institute of Biotechnology. PITC was dissolved in DMSO to prepare stock solutions for in vitro assays and stored at $-20^{\circ} \mathrm{C}$. Cells in the control groups were treated with vehicle at an equal volume. To minimize toxicity, DMSO concentration was $>0.1 \%$ for cell culture. Anti-poly-ADP-ribose polymerase (PARP; catalog no. 9542; 1:1,000), anti-cleaved caspase-3 (catalog no. 9662; 1:1,000), anti-cleaved caspase-9 (catalog no. 9502; 1:1,000), anti-GAPDH (catalog no. 5174; 1:1,000), anti-cytochrome c (Cyt c; catalog no. 4272; 1:1,000), anti-p53 (catalog no. 2527; 1:1,000) and anti-phosphorylated p53 (p-p53; catalog no. 2521; 1:1,000) antibodies were purchased from Cell Signaling Technology, Inc. Anti-cyclin A1 antibody (catalog no. ab118897; 1:1,000) was obtained from Abcam, Inc. Antibodies against Bcl-2 (catalog no. A2845; 1:1,000), Bax (catalog no. A0207; 1:1,000) and $\beta$-tubulin (catalog no. AC008; 1:1,000 for western blot analysis and 1:400 for immunofluorescence assay) were purchased from ABclonal Biotech Co., Ltd.

Cells and cell culture. Human GC MGC-803 and HGC-27 cell lines were purchased from the Shanghai Institute of Cell Biology, Chinese Academy of Sciences, and cultured in RPMI-1640 (Gibco; Thermo Fisher Scientific, Inc.) supplemented with $10 \%$ fetal bovine serum (Gibco; Thermo Fisher Scientific, Inc.) and $100 \mu \mathrm{g} / \mathrm{ml}$ streptomycin and $100 \mathrm{U} / \mathrm{ml}$ penicillin (HyClone; GE Healthcare Life Sciences) in a $37^{\circ} \mathrm{C}$ incubator with a humidified atmosphere and $5 \% \mathrm{CO}_{2}$.

Cell viability assay. The effects of PITC on GC cells were evaluated by Cell Counting Kit-8 [CCK-8; Yeasen Biotechnology (Shanghai) Co., Ltd.]. Prior to treatment, MGC-803 and HGC-27 cells were plated in 96-well plates with 1,500 cells per well and incubated at $37^{\circ} \mathrm{C}$ overnight. MGC- 803 cells were treated with PITC at concentrations of $0,50,100,150,200$ and $250 \mu \mathrm{M}$, whereas HGC-27 cells were treated with PITC at $0,20,30,40,50$ and $60 \mu \mathrm{M}$ for 24,48 and $72 \mathrm{~h}$ at $37^{\circ} \mathrm{C}$. CCK-8 working solution was prepared with $10 \mu \mathrm{l}$ CCK-8 solution and $100 \mu \mathrm{l}$ culture medium; culture medium was replaced with $100 \mu \mathrm{l}$ working solution in each well and incubated for $2 \mathrm{~h}$ at $37^{\circ} \mathrm{C}$. The absorbance at $450 \mathrm{~nm}$ was measured by a microplate reader (Bio-Rad Laboratories, Inc.).

Colony formation assay. A total of 500 cells/well were seeded in 6-well plates and treated with PITC $(0,50,100$ and $150 \mu \mathrm{M}$ for MGC-803; 0, 20, 40 and $60 \mu \mathrm{M}$ for HGC-27) for $48 \mathrm{~h}$ at $37^{\circ} \mathrm{C}$ and allowed to form colonies in fresh medium for 10 days. During this time, culture medium was refreshed every three days. Subsequently, the plates were washed gently with PBS and fixed with 4\% paraformaldehyde for $15 \mathrm{~min}$ and stained with $0.1 \%$ crystal violet (Sigma-Aldrich) for $15 \mathrm{~min}$ at room temperature. PBS was used to remove the excess crystal violet, and images of the plates were captured. Colonies with $\geq 50$ cells were counted.

Apoptosis assay. FITC Annexin V Apoptosis Detection Kit I (BD Biosciences) was used to detect apoptosis according to the manufacturer's instructions. MGC-803 and HGC-27 cells were plated in 6 -well plates with $8 \times 10^{4}$ cells per well and treated with PITC for $48 \mathrm{~h}$ at $37^{\circ} \mathrm{C}$ following adherence. Floating and adherent cells were collected and resuspended in
$100 \mu \mathrm{l}$ binding buffer which containing $5 \mu \mathrm{l}$ FITC-conjugated annexin- $\mathrm{V}$ and $5 \mu \mathrm{l}$ propidium iodide (PI), then incubated for $15 \mathrm{~min}$ at room temperature. After which, $400 \mu \mathrm{l}$ binding buffer was added to the suspension and then immediately analyzed by flow cytometry (BD Biosciences).

Cell cycle analysis. MGC-803 and HGC-27 cells were treated with PITC at different concentrations for $48 \mathrm{~h}$. The cells were harvested, washed with cold PBS and fixed with cold $70 \%$ ethanol at $-20^{\circ} \mathrm{C}$ overnight. Each sample contained $\sim 2 \times 10^{5}$ cells was washed with cold PBS, resuspended in $250 \mu \mathrm{l}$ staining solution containing $10 \mathrm{mg} / \mathrm{ml}$ of RNase and $1 \mathrm{mg} / \mathrm{ml}$ propidium iodide (Sigma-Aldrich; Merck KGaA) and incubated at $37^{\circ} \mathrm{C}$ for $30 \mathrm{~min}$ in the dark. Cell cycle of all samples was analyzed by flow cytometry (BD Biosciences).

Intracellular reactive oxygen species detection. A ROS Assay kit (Beyotime Institute of Biotechnology) was used to determine the level of intracellular ROS. Considering that the production of ROS must be earlier than the presence of cell apoptosis and cell cycle arrest, $24 \mathrm{~h}$ was selected as the treatment time in this assay. MGC-803 and HGC-27 cells were seeded in 6-well plates at a density of $8 \times 10^{4}$ cells per well and treated with PITC $(0,50$ and $150 \mu \mathrm{M}$ for MGC-803; 0, 20 and $60 \mu \mathrm{M}$ for $\mathrm{HGC}-27$ ) for $24 \mathrm{~h}$ at $37^{\circ} \mathrm{C}$. The following steps were performed according to the manufacturer's protocol. Briefly, adherent cells were washed with PBS, and each well was incubated with $1 \mathrm{ml} 10 \mathrm{mM}$ dichloro-dihydro-fluorescein diacetate (DCFH-DA) at $37^{\circ} \mathrm{C}$ for $25 \mathrm{~min}$. The wells were washed three times with serum-free RPMI-1640 medium. The ROS in situ was observed under a fluorescence microscope (Leica Microsystems, Inc).

To evaluate the intracellular ROS with flow cytometry, the cells were collected by trypsinization prior to incubation with DCFH-DA. Samples were agitated every 3 min during incubation and immediately analyzed by flow cytometry.

Comet assay. The comet assay, also known as single cell gel electrophoresis (SCGE), was performed according to the protocol described by Speit et al (15) with minor modifications. Briefly, following $24 \mathrm{~h}$ treatment with PITC, MGC-803 and HGC-27 cells were harvested and washed with PBS, and cell viability was determined by trypan blue. After incubation with trypan blue for $5 \mathrm{~min}$ at room temperature, the cells were counted under light microscope and living cells were those without being stained by trypan blue. A sample with $<5 \%$ dead cells was considered acceptable. Normal melting agarose (1\%) was prepared to form the bottom layer of the gel. The following steps were performed in the dark. A total of $10 \mu \mathrm{l}$ cell suspensions containing $1 \times 10^{4}$ cells were mixed with $90 \mu \mathrm{l}$ $0.5 \%$ low melting point agarose and distributed on slides. Following solidification, cells were lysed in cold, freshly made lysing solution (2.5 M NaCl, 0.1 M EDTA, 0.01 M Tris, 1\% Triton $\mathrm{X}-100,10 \% \mathrm{DMSO}, \mathrm{pH}=10$ ) at $4^{\circ} \mathrm{C}$ for $1.5 \mathrm{~h}$. The slides were placed in an electrophoresis tank filled with electrophoresis buffer (0.001 $\mathrm{M} \mathrm{Na}_{2}$ EDTA, $0.3 \mathrm{M} \mathrm{NaOH}, \mathrm{pH}=13$ ) for $20 \mathrm{~min}$, and electrophoresis was performed for $25 \mathrm{~min}$ at $25 \mathrm{~V}$ in an ice bath. The slides were set in a neutralization buffer for $15 \mathrm{~min}$ at room temperature, which was replaced for every 5 min. 4',6-diamidino-2-phenylindole (Beyotime Institute of 
Biotechnology) was used as a nucleic acid stain to identify the DNA tracks. The comets were magnified 100 times and detected with a fluorescence microscope and Leica Application Suite software (version 4.2, Leica Microsystems, Inc.). For each slide, five fields were counted and each experiment was performed in triplicate.

Intracellular GSH/glutathione disulfide (GSSG) level detection. GSH and GSSG Assay kit (catalog no. S0053; Beyotime Institute of Biotechnology) was used to detect the level of GSH/GSSG in MGC-803 and HGC-27 cells. Following treatment with PITC $(0,50$ and $150 \mu \mathrm{M}$ for MGC-803; 0, 20 and $60 \mu \mathrm{M}$ for HGC-27) for $12 \mathrm{~h}$ at $37^{\circ} \mathrm{C}$, the cells were harvested and counted. Cells were transferred to new tubes to ensure equal the cell numbers in each group and washed with PBS. The protein removal solution was used to resuspend cells. The tubes were placed in liquid nitrogen and $37^{\circ} \mathrm{C}$ water twice for fast freezing and thawing to split the cell membrane and subsequently placed in $4^{\circ} \mathrm{C}$ for $5 \mathrm{~min}$ and centrifuged at $10,000 \mathrm{xg}$ at $4^{\circ} \mathrm{C}$ for $10 \mathrm{~min}$. The supernatants were collected to detect the amount of total (t)GSH. GSH and GSSG Assay kit (Beyotime Institute of Biotechnology) was used to detect tGSH following the manufacturer's instructions. Briefly, GSH assay working solution and samples were added to 96-well plates and incubated at room temperature for $5 \mathrm{~min}$; reduced nicotinamide adenine dinucleotide phosphate was added into the reactive system and incubated for $25 \mathrm{~min}$ at room temperature. Absorbance at $412 \mathrm{~nm}$ was measured by a microplate reader.

Cellular immunofluorescence staining. Cellular immunofluorescence staining was conducted to detect the expression of $\beta$-Tubulin. A total of $4 \times 10^{4}$ of MGC-803 and HGC-27 cells were seeded in 12-well plates, which were previously laid with sterile cover glasses and cultured for $24 \mathrm{~h}$ at $37^{\circ} \mathrm{C}$ until the cells adhered to the glass slides. The cells were then treated with PITC $(60 \mu \mathrm{M})$, DMSO or PITC $(60 \mu \mathrm{M})$ and NAC $(5 \mu \mathrm{M})$ respectively for $12 \mathrm{~h}$ at $37^{\circ} \mathrm{C}$. Cold $4 \%$ paraformaldehyde was used to fix cells at $4^{\circ} \mathrm{C}$ for $20 \mathrm{~min}, 0.2 \%$ Triton-X was used to perforate cell membrane for $10 \mathrm{~min}$, and cells were blocked with $1 \%$ bovine serum albumin (Gibco; Thermo Fisher Scientific, Inc.) for $1 \mathrm{~h}$ at room temperature. Subsequently, cells were incubated with antibody against $\beta$-tubulin (1:200) at $4^{\circ} \mathrm{C}$ overnight. Following incubation with the primary antibodies, cells were then incubated with donkey anti-rabbit IgG HRP-conjugated fluorescent secondary antibody (1:200; catalog no. 34206ES60; Yeasen Biotechnology, Co., Ltd.) for $30 \mathrm{~min}$ and the nuclei were stained with DAPI for $5 \mathrm{~min}$ at room temperature. Finally, the expression and morphology of $\beta$-tubulin were detected by fluorescence microscopy at $\mathrm{x} 400$ magnification.

Western blot analysis. Cells were treated with PITC at various concentrations for $24 \mathrm{~h}(0,50,100$ and $150 \mu \mathrm{M}$ for MGC-803; 0, 20, 40 and $60 \mu \mathrm{M}$ for HGC-27) and washed twice with cold PBS prior to protein extraction. Radio Immunoprecipitation Assay (RIPA) buffer (Beyotime Institute of Biotechnology) containing $1 \%$ protease inhibitor cocktail (Beyotime Institute of Biotechnology) was used to lyse cells, which were then removed from the dishes and continued to be lysed on ice for $30 \mathrm{~min}$. The extractions were centrifuged at $14,000 \mathrm{x} \mathrm{g}$ at $4^{\circ} \mathrm{C}$ for $15 \mathrm{~min}$, and the concentration was determined by a bicinchoninic acid (BCA) assay kit (Beyotime Institute of Biotechnology) according to the manufacturer's instructions. Western blot analysis was conducted to detect the expression of proteins as previously described (16). Briefly, an equal amount $(10 \mu \mathrm{g})$ of proteins was separated by SDS-PAGE (10\% gel) and transferred to PVDF membranes. Subsequently, the PVDF membranes were blocked with 5\% skimmed milk and probed with primary antibodies against PARP, $\beta$-tubulin, p-53, p-p53, cleaved caspase-9, cleaved caspase-3, Bcl-2, Bax, cyclin A1 and Cyt c $(1: 1,000)$ at $4^{\circ} \mathrm{C}$ overnight and further incubated with HRP-conjugated secondary antibodies at room temperature for $1 \mathrm{~h}$. The bands were visualized by chemiluminescence (EMD Millipore) and Image $\mathbf{J}$ software (version 1.48; National Institues of Health) was used to quantify the densitometric values of the detected bands. GAPDH was used as an endogenous control.

Statistical analysis. All values are presented as the mean \pm SD and were analyzed using GraphPad Prism 5 (GraphPad Software, Inc.) and IBM SPSS Statistics 24 (IBM Corp.). Student's t-test was used to compare the differences between two groups, and multiple comparisons of means were conducted using one-way analysis of variance with Tukey's Honestly Significant Difference. $\mathrm{P}<0.05$ was considered to indicate a statistically significant difference.

\section{Results}

PITC inhibits GC cell proliferation and viability. Cell proliferation and viability were analyzed using CCK-8 and colony formation assays. Treatment with PITC inhibited the viability of MGC-803 and HGC-27 cells; the inhibitory effect grew as the concentration and treatment time of PITC increased (Fig. 1B). The half maximal inhibitory concentration $\left(\mathrm{IC}_{50}\right)$ values of MGC-803 and HGC-27 cells at $48 \mathrm{~h}$ were $\sim 100$ and $\sim 40 \mu \mathrm{M}$, respectively. According to the curves, 50, 100 and $150 \mu \mathrm{M}$ were selected as the treatment concentrations for the MGC-803 cell line, whereas 20,40 and $60 \mu \mathrm{M}$ were selected for the HGC-27 cell line in subsequent experiments. The results of the colony formation assay indicated that PITC repressed the ability of GC cells to form colonies, which was associated with the drug concentrations (Fig. 1C and D). These results suggested that PITC may significantly affect gastric cancer cell viability and proliferation.

PITC induces apoptosis and cell cycle arrest in GC cells. To further demonstrate whether PITC induces cell cycle arrest and apoptosis, the effects of PITC on MGC-803 and HGC-27 cells were evaluated by flow cytometry. The number of apoptotic cells, including early and late apoptosis, were apparently increased in a dose-dependent manner following PITC treatment (Fig. 2A and B). The results presented in Fig. 2C-E demonstrated that PITC increased the percentage of cells in the S-phase compared with the control groups and decreased the expression of Cyclin A1. These results suggested that apoptosis and cell cycle arrest induced by PITC may serve a vital role in the inhibition of cell viability and proliferation. 

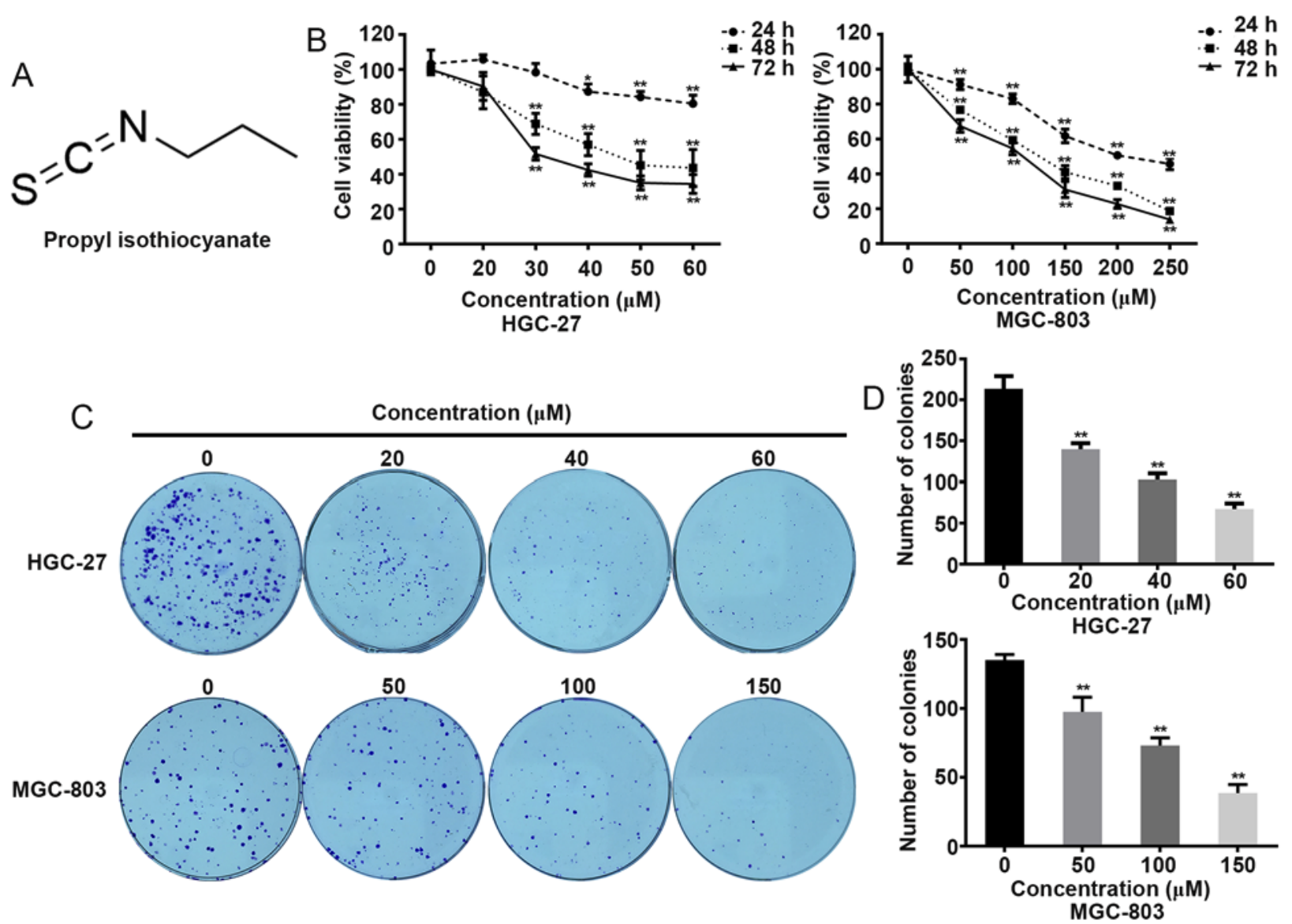

Figure 1. PITC inhibits the proliferation and viability of gastric cancer cells. (A) The chemical structure of PITC. (B) MGC-803 and HGC-27 cells were treated with PITC at different concentrations for 24, 48 and $72 \mathrm{~h}$, and the cell viability was detected by Cell Counting Kit-8 assay. (C and D) MGC-803 and HGC-27 cells were treated with various concentrations of PITC for $48 \mathrm{~h}$ and cultured in fresh medium for 10 days to form colonies. Colonies were stained and counted 10 days after treatment. Each bar represents the mean $\pm \mathrm{SD}$ from three independent experiments. ${ }^{*} \mathrm{P}<0.05,{ }^{* *} \mathrm{P}<0.01$ vs. $0 \mu \mathrm{M}$ PITC. PITC, propyl isothiocyanate.

PITC reduces $t G S H$ and increases $R O S$ production in $G C$ cells and causes DNA damage. The results of the present study demonstrated that the levels of tGSH were decreased in PITC-treated groups compared with the control groups (Fig. 3A). Simultaneously, an apparent increase in ROS levels was detected by fluorescence microscopy (Fig. 3B). SCGE was performed to examine the effects of PITC on DNA; the results suggested that PITC may induce DNA damage in the two GC cell lines (Fig. 3C), and higher doses of PITC could lead to longer tails of comets which means more severe damage of DNA. Therefore, treatment with PITC may lead to the accumulation of ROS in the two GC cell lines and subsequently induce DNA damage.

PITC affects the signaling pathway of caspase and Bcl-2 family members. Apoptosis is a physiological process triggered by several pathways. Mitochondria-dependent apoptosis is one of these pathways which is initiated by Cyt c (17). To confirm the involvement of mitochondria in PITC-induced apoptosis, a number of apoptosis-related proteins were analyzed by western blotting. As demonstrated in Fig. 3D, PITC significantly increased the expression of Cyt c, cleaved caspase-9, cleaved caspase- 3 and cleaved PARP in a dose-dependent manner. The expression of p-p53, which is activated by DNA damage, was upregulated and total p53 was downregulated; in addition, Bax expression was upregulated and $\mathrm{Bcl}-2$ expression was downregulated. Therefore, the dysfunction of mitochondria and DNA damage may be associated with PITC-induced apoptosis.

$N A C$ rescues the effects of PITC on apoptosis of GC cells. To investigate whether the effect of PITC on the intracellular redox balance is the cause of apoptosis, reversion tests with NAC were performed. Following co-treatment with PITC and NAC, the levels of ROS were analyzed by flow cytometry, which demonstrated that the increase of ROS in PITC-treated groups were downregulated by NAC (Fig. 4A). In addition, adding NAC reversed the viability inhibition by PITC and decreased the percentage of apoptotic cells compared with the groups treated with PITC only (Fig. 4B-D). The results of the western blotting also suggested that NAC may rescue the changes of several apoptosis-related proteins induced by PITC (Fig. 4E). In summary, these results indicated that the accumulation of ROS induced by PITC triggers apoptosis of MGC-803 and HGC-27 cells.

PITC induces $\beta$-tubulin degradation in gastric cancer cells. In order to confirm the effect of PITC on $\beta$-tubulin of MGC-803 and HGC-27 cells, the present study performed cellular immunofluorescence staining. As a result, the treatment of PITC decreases the expression level of $\beta$-tubulin both in MGC-803 and HGC-27 cells (Fig. S1A), and the consequence 

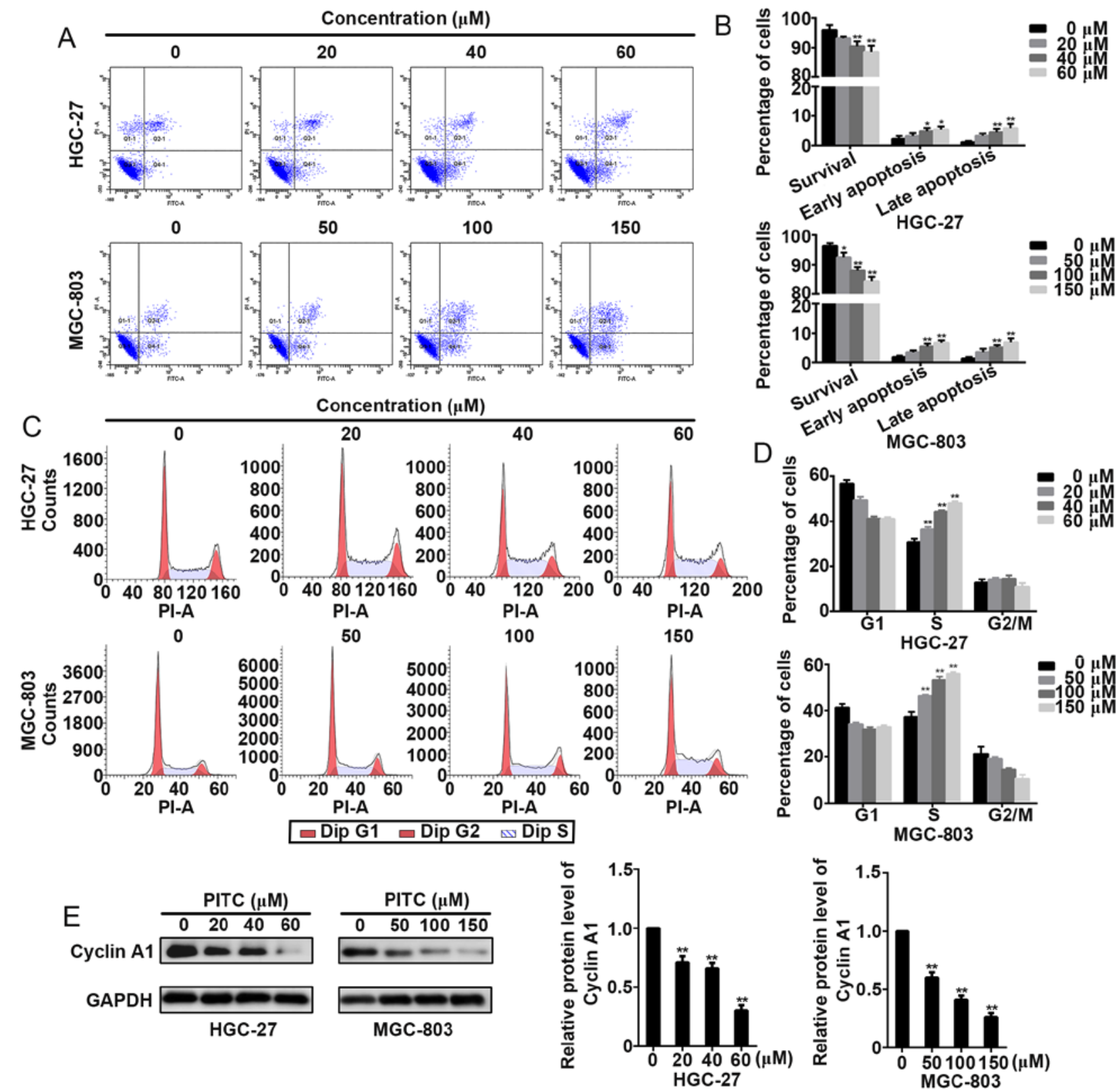

Figure 2. PITC induces S-phase arrest and apoptosis in gastric cancer cells. MGC-803 and HGC-27 cells were pretreated with PITC at different concentrations for $48 \mathrm{~h}$. (A and B) Cells were stained with Annexin V-FITC/PI, and the percentages of apoptotic cells were analyzed by flow cytometry. (B) Data are expressed as the mean $\pm \mathrm{SD}$ of three independent experiments. (C and D) The cell cycle distribution was determined by flow cytometry. (D) Data are presented as the mean \pm SD of three independent experiments. (E) The expression levels of Cyclin A1 were detected by western blotting. GAPDH was used as a loading control. The results are expressed as fold-change relative to the controls. ${ }^{*} \mathrm{P}<0.05$ and ${ }^{* *} \mathrm{P}<0.01$ vs. $0 \mu \mathrm{M}$ PITC. PITC, propyl isothiocyanate; PI, propidium iodide.

was supported by the results of the western blot analysis in Fig. S1B. Furthermore, the effect of PITC on $\beta$-tubulin was reversed by NAC (Fig. S1C). These findings indicated that PITC induces the degradation of $\beta$-tubulin in gastric cancer cells.

\section{Discussion}

ITCs are a category of compounds extracted from cruciferous plants that exhibit versatile biological activities. The anticancer activities of PITC have not been reported to date. In the present study, the effects of PITC on the biological behavior of GC cells were investigated, and the relevant molecular mechanisms were explored. The results demonstrated that PITC inhibited the viability of MGC-803 and HGC-27 cells. HGC-27 cells exhibited higher sensitivity to PITC compared with that of MGC-803 cells, the reason of which is unknown and requires further study. The underlying mechanisms were preliminarily studied by flow cytometry; the results demonstrated significant cell cycle arrest at the S-phase and apoptosis induction, which suggested the potential of PITC to suppress cancer cell proliferation. The mechanisms of PITC-induced apoptosis were subsequently explored.

GSH is a metabolite involved in the maintenance of redox homeostasis in cells; the balance of ROS is disturbed when GSH is dysregulated (18). According to previous studies, the 

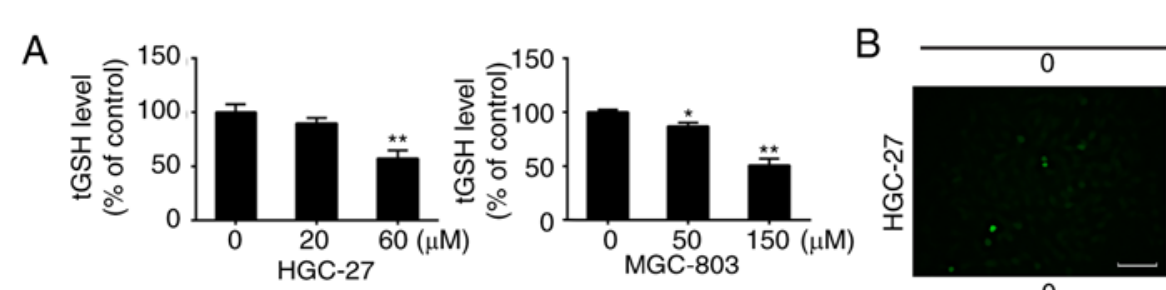

Concentration $(\mu \mathrm{M})$

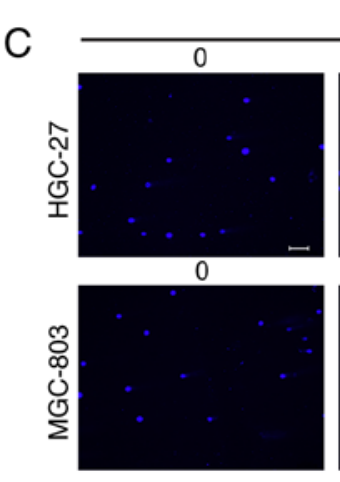

Concentration $(\mu \mathrm{M})$

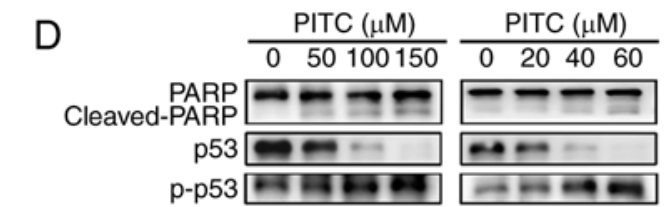

Cleaved caspase-9

$\mathrm{Bcl}-2 \backsim-\ldots-\ldots$

$\mathrm{Bax}-\infty-$

Cleaved caspase-3 $\because-m-$

Cytochrome $\mathrm{C} \longrightarrow$

GAPDH $\underset{\text { MGC-803 }}{\longrightarrow-\infty-}$
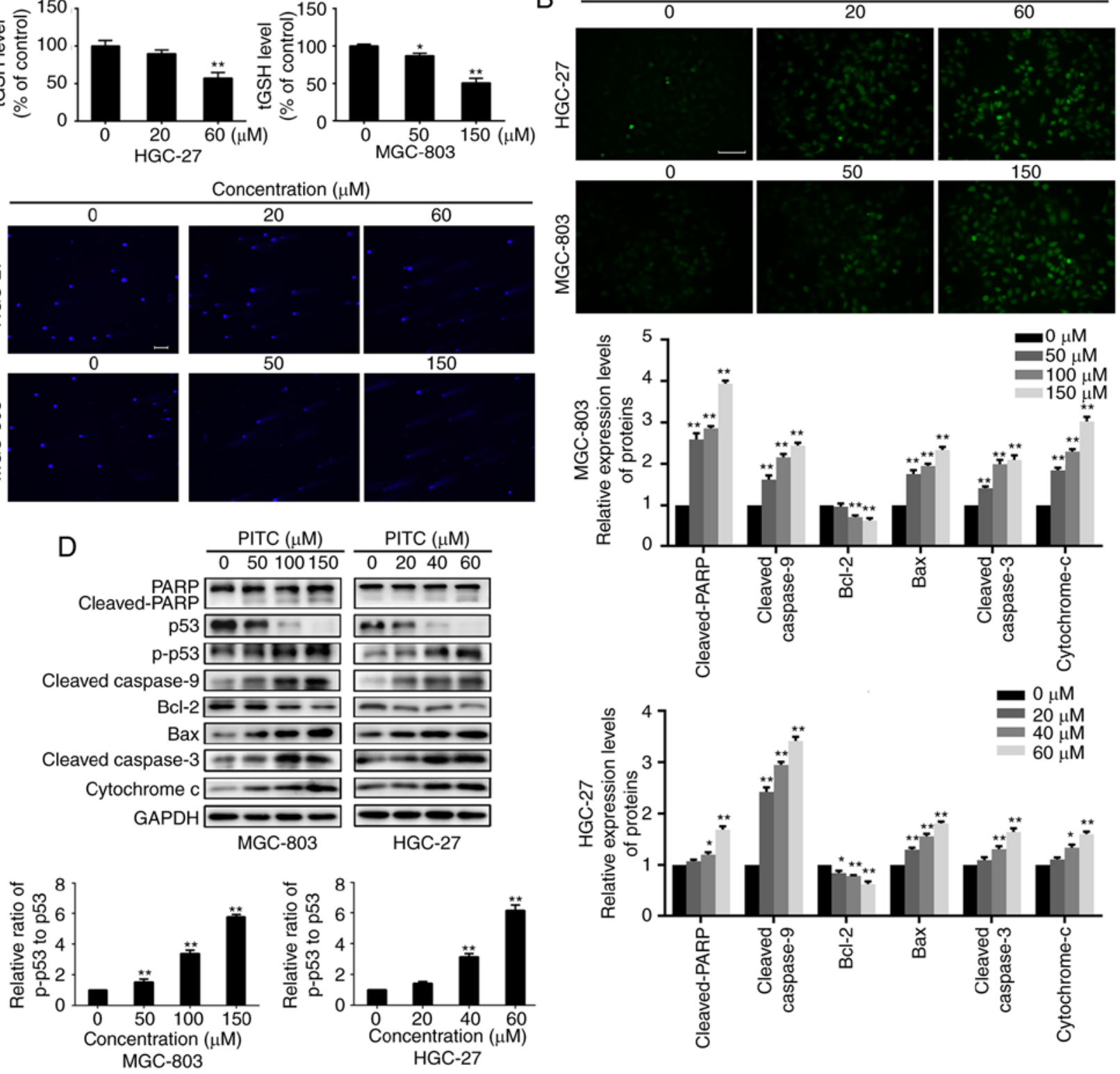

Figure 3. PITC induces GSH depletion, ROS accumulation and DNA damage in gastric cancer cells. (A) MGC-803 and HGC-27 cells were pretreated with PITC for $12 \mathrm{~h}$; the results of the analysis of tGSH levels are presented as the mean \pm SD of three independent experiments. (B) The intracellular levels of ROS at $24 \mathrm{~h}$ of PITC treatment were observed. Green fluorescence representing intracellular ROS was analyzed using fluorescence microscopy. Scale bar, $100 \mu \mathrm{m}$. (C) DNA damage at $24 \mathrm{~h}$ of PITC treatment of MGC-803 and HGC-27 cells was detected by the Comet assay and observed with a fluorescence microscope. Different-sized tails indicated the level of DNA damage. Scale bar, $100 \mu \mathrm{m}$. (D) Western blotting was performed to analyze the expression levels of apoptosis-related proteins, and GAPDH was used as a loading control. The results are expressed as fold-change relative to the controls. ${ }^{*}<0.05$ and ${ }^{* *} \mathrm{P}<0.01$ vs. $0 \mu \mathrm{M}$ PITC. PITC, propyl isothiocyanate; GSH, glutathione; tGSH, total GSH; ROS, reactive oxygen species.

anticancer activities of ITCs may be associated with their direct and indirect interactions with cellular components. Conjugation with GSH is the first step in ITCs metabolism, which consumes the natural anti-oxidant and possibly influences the intracellular redox status $(8,19)$. After binding with GSH, ITCs will then be excreted from the cytosol, which may cause the depletion of cytosolic GSH $(5,20,21)$. In the present study, the intracellular tGSH levels were measured, and a concentration-dependent decrease of tGSH following PITC treatment was revealed, consistent with the increase in ROS. DNA damage in MGC-803 and HGC-27 cells, which is one of the consequences of increased ROS levels $(22,23)$, was also observed in the present study. The results suggested that the accumulation of ROS, which may be induced by GSH depletion, resulted in PITC-induced apoptosis in MGC-803 and HGC-27 cells.

To further investigate the mechanisms of PITC-induced apoptosis, the damage induced by excessive ROS was considered. Proteins, lipids, DNA and other intracellular components are targets of ROS (24). Mitochondria can be damaged by excessive ROS through direct oxidation of proteins and lipids, leading to changes in their structure and function (25). As the permeability of the mitochondrial membrane increases, Cyt $\mathrm{c}$ is released into the cytosol and triggers mitochondria-dependent apoptosis by activating caspase $(25,26)$. Therefore, it may be speculated that PITC-induced apoptosis of MGC-803 and HGC-27 cells may be mediated by the mitochondria. In the 

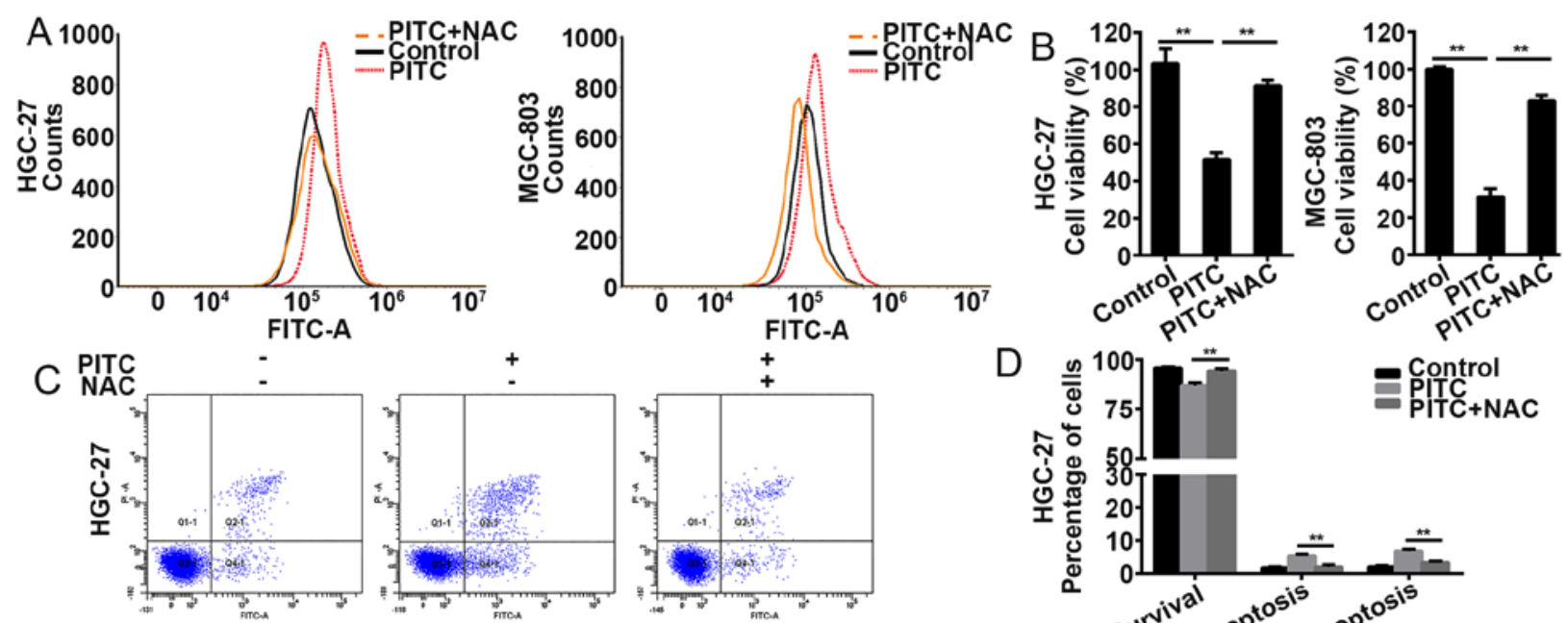

$$
\text { D }
$$
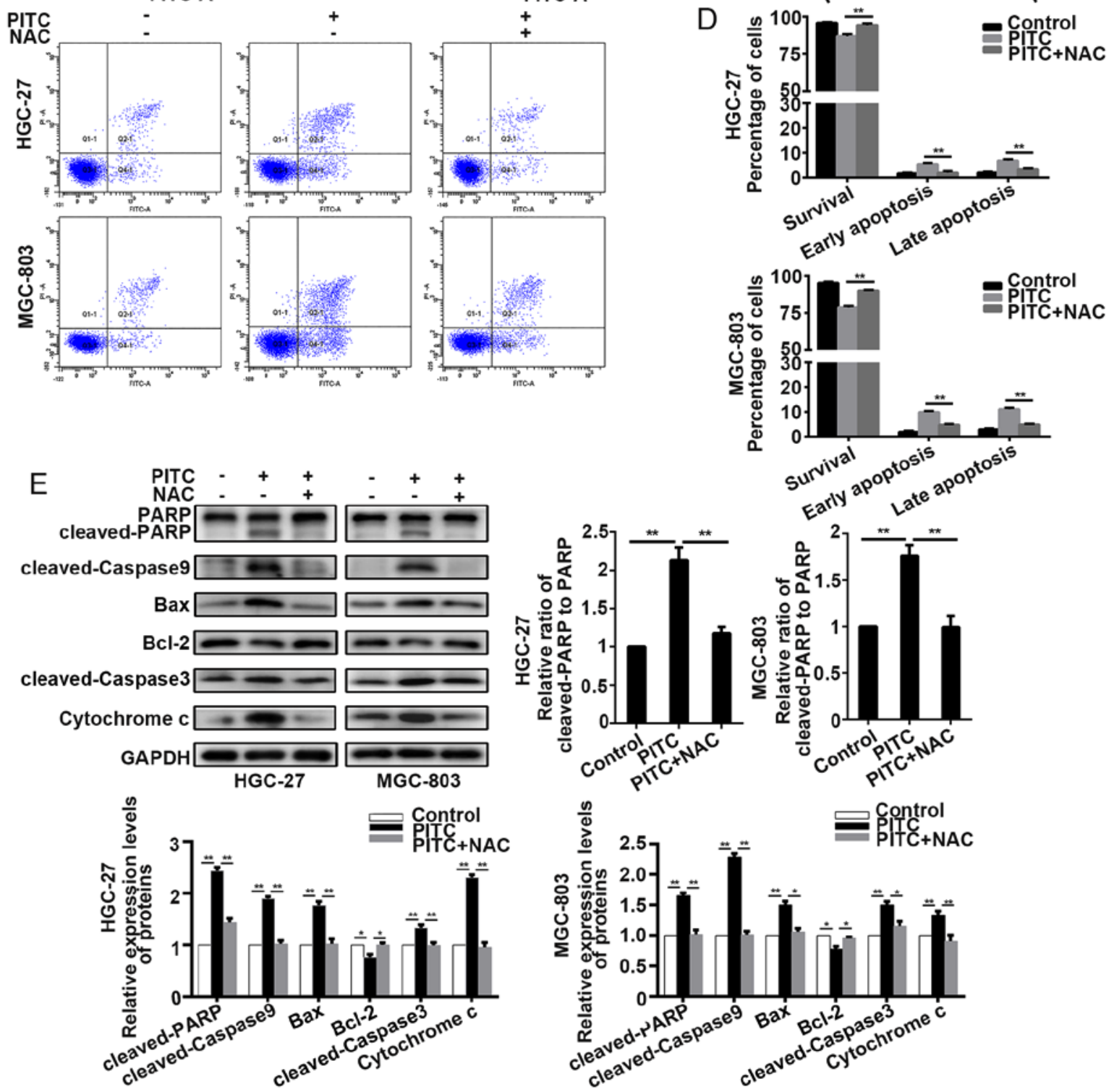

Figure 4. NAC reverses the effects of PITC in gastric cancer cells. MGC-803 and HGC-27 cells were pretreated with DMSO, PITC (150 $\mu$ M for MGC-803 and $60 \mu \mathrm{M}$ for HGC-27 cells) or a combination of PITC and $5 \mu \mathrm{M}$ NAC. (A) The levels of ROS were measured by flow cytometry. (B) Cell viability was detected by Cell Counting Kit-8 assay. (C and D) Treated cells were stained with Annexin V-FITC/propidium iodide and analyzed by flow cytometry. (D) The percentage of apoptotic cells are presented as the mean \pm SD of three independent experiments. (E) Western blotting was performed to analyze the expression levels of apoptosis-related proteins, and GAPDH was used as a loading control. The results are expressed as fold-change relative to the controls. ${ }^{*} \mathrm{P}<0.05$ and ${ }^{* *} \mathrm{P}<0.01$. PITC, propyl isothiocyanate; N-acetyl-L-cysteine; ROS, reactive oxygen species.

present study, the exposure to PITC increased the expression of Cyt c, induced the upregulation of active forms of caspase-3 and -9 , and led to the activation of PARP. In addition, other targets of ROS contribute to apoptosis. ROS-induced DNA damage promotes the phosphorylation of $\mathrm{p} 53$ by activating ataxia telangiectasia-mutated kinase and subsequently regulates the expression of downstream proteins, including the $\mathrm{Bcl}-2$ family (27). In the present study, PITC increased the ratio of p-p53/total p53 and the expression of Bax, as well as induced the downregulation of Bcl-2 in MGC-803 and HGC-27 cells. These results suggested that ROS-induced mitochondrial dysfunction and DNA damage may mediate PITC-induced apoptosis.

The effects of PITC were rescued by the ROS scavenger NAC, although they were not fully reversed, which suggests that there may be other ways for PITC to induce apoptosis. $\beta$-tubulin is a potential target of ITCs due to being rich in cysteine 
residues (28). The results of the present study demonstrated that the degradation of $\beta$-tubulin was promoted following treatment with PITC. This effect was partially attenuated by NAC, which may serve as a competitor of $\beta$-tubulin to bind PITC.

In summary, the results of the present study indicate that PITC may exhibit potent anticancer activities in GC cell lines by promoting apoptosis and inducing cell cycle arrest. PITC may trigger apoptosis by inducing GSH depletion, leading to the accumulation of ROS, mitochondrial dysfunction and DNA damage. Mitochondria-associated apoptosis and DNA damage are the possible mechanisms mediating these effects in the two studied GC cell lines. To explore the efficacy and safety of PITC in vivo, further research is required using xenograft tumor models.

\section{Acknowledgements}

Not applicable.

\section{Funding}

The present study was funded by the National Natural Science Foundation of China (grant no. 81572819) and the Shanghai Key Laboratory of Biliary Tract Disease Research Foundation (grant no. 17DZ2260200).

\section{Availability of data and materials}

The datasets used and/or analyzed during the present study are available from the corresponding author upon reasonable request.

\section{Author contributions}

JG and GFH conceived the study. LH, CC and WD collected and analyzed the data and finalized the manuscript. LH and CC performed the experiments. JHL assessed the data and revised the manuscript. JG and GFH supervised and managed the project. All authors have approved the final manuscript.

\section{Ethics approval and consent to participate}

Not applicable.

\section{Patient consent for publication}

Not applicable.

\section{Competing interests}

The authors declare that they have no competing interests.

\section{References}

1. Chen W, Zheng R, Baade PD, Zhang S, Zeng H, Bray F, Jemal A Yu XQ and He J: Cancer statistics in China, 2015. CA Cancer J Clin 66: 115-132, 2016.

2. Yang L, Zheng R, Wang N, Yuan Y, Liu S, Li H, Zhang S, Zeng H and Chen W: Incidence and mortality of stomach cancer in China, 2014. Chin J Cancer Res 30: 291-298, 2018.

3. Siegel RL, Miller KD and Jemal A: Cancer statistics, 2018. CA Cancer J Clin 68: 7-30, 2018
4. Van Cutsem E, Sagaert X, Topal B, Haustermans K and Prenen H: Gastric cancer. Lancet 388: 2654-2664, 2016.

5. Beran F, Sporer T, Paetz C, Ahn SJ, Betzin F, Kunert G, Shekhov A, Vassão DG, Bartram S, Lorenz S and Reichelt M: One pathway is not enough: The cabbage stem flea beetle psylliodes chrysocephala uses multiple strategies to overcome the glucosinolate-myrosinase defense in its host plants. Front Plant Sci 9: 1754, 2018.

6. Brown KK and Hampton MB: Biological targets of isothiocyanates. Biochim Biophys Acta 1810: 888-894, 2011

7. Paul S, Geng CA, Yang TH, Yang YP and Chen JJ: Phytochemical and health-beneficial progress of turnip (Brassica rapa). J Food Sci 84: 19-30, 2019.

8. Mi L, Di Pasqua AJ and Chung FL: Proteins as binding targets of isothiocyanates in cancer prevention. Carcinogenesis 32: 1405-1413, 2011

9. Zhang $\mathrm{C}$, Wu H, Zhao Y, Ma Z and Zhang X: Comparative studies on mitochondrial electron transport chain complexes of Sitophilus zeamais treated with allyl isothiocyanate and calcium phosphide. Pestic Biochem Physiol 126: 70-75, 2016.

10. Zhang T and Chen W: The Candida albicans inhibitory activity of the extract from papaya (Carica papaya L.) seed relates to mitochondria dysfunction. Int J Mol Sci 18: pii: E1858, 2017.

11. Sita G, Hrelia P, Graziosi A and Morroni F: Sulforaphane from cruciferous vegetables: Recent advances to improve glioblastoma treatment. Nutrients 10: pii: E1755, 2018.

12. Bo P, Lien JC, Chen YY, Yu FS, Lu HF, Yu CS, Chou YC, Yu CC and Chung JG: Allyl isothiocyanate induces cell toxicity by multiple pathways in human breast cancer cells. Am J Chin Med 44: 415-437, 2016.

13. Huang YP, Jiang YW, Chen HY, Hsiao YT, Peng SF, Chou YC, Yang JL, Hsia TC and Chung JG: Benzyl isothiocyanate induces apoptotic cell death through mitochondria-dependent pathway in gefitinib-resistant NCI-H460 human lung cancer cells in vitro. Anticancer Res 38: 5165-5176, 2018.

14. Chou YC, Chang MY, Lee HT, Shen CC, Harnod T, Liang YJ, Wu RS, Lai KC, Hsu FT and Chung JG: Phenethyl isothiocyanate inhibits in vivo growth of xenograft tumors of human glioblastoma cells. Molecules 23: pii: E2305, 2018.

15. Speit $G$ and Rothfuss A: The comet assay: A sensitive genotoxicity test for the detection of DNA damage and repair. Methods Mol Biol 920: 79-90, 2012.

16. Xiang SS, Wang XA, Li HF, Shu YJ, Bao RF, Zhang F, Cao Y, Ye YY, Weng H, Wu WG, et al: Schisandrin B induces apoptosis and cell cycle arrest of gallbladder cancer cells. Molecules 19: 13235-13250, 2014.

17. Zhang H, Kou Y, Li J, Chen L, Mao Z, Han XX, Zhao B and Ozaki Y: Nickel nanowires combined with surface-enhanced raman spectroscopy: Application in label-free detection of cytochrome c-mediated apoptosis. Anal Chem 91: 1213-1216, 2019.

18. Tang JY, Farooqi AA, Ou-Yang F, Hou MF, Huang HW, Wang HR, Li KT, Fayyaz S, Shu CW and Chang HW: Oxidative stress-modulating drugs have preferential anticancer effects-involving the regulation of apoptosis, DNA damage, endoplasmic reticulum stress, autophagy, metabolism, and migration. Semin Cancer Biol, Aug 24, 2018 (Epub ahead of print).

19. Øverby A, Stokland RA, Åsberg SE, Sporsheim B and Bones AM: Allyl isothiocyanate depletes glutathione and upregulates expression of glutathione S-transferases in Arabidopsis thaliana. Front Plant Sci 6: 277, 2015.

20. Kim YJ, Lee DH, Ahn J, Chung WJ, Jang YJ, Seong KS, Moon JH, Ha TY and Jung CH: Pharmacokinetics, tissue distribution, and anti-lipogenic/adipogenic effects of allyl-isothiocyanate metabolites. PLoS One 10: e0132151, 2015.

21. Zhang Y, Yao S and Li J: Vegetable-derived isothiocyanates: Anti-proliferative activity and mechanism of action. Proc Nutr Soc 65: 68-75, 2006.

22. Yun M, Choi AJ, Lee YC, Kong M, Sung JY, Kim SS and Eun YG: Carbonyl reductase 1 is a new target to improve the effect of radiotherapy on head and neck squamous cell carcinoma. J Exp Clin Cancer Res 37: 264, 2018.

23. Kivisaar M: The effect of cellular redox status on the evolvability of new catabolic pathways. MBio 9: pii: e01981-18, 2018.

24. Radi R: Oxygen radicals, nitric oxide, and peroxynitrite: Redox pathways in molecular medicine. Proc Natl Acad Sci USA 115: 5839-5848, 2018. 
25. Piantadosi CA and Suliman HB: Redox regulation of mitochondrial biogenesis. Free Radic Biol Med 53: 2043-2053, 2012.

26. Sinha K, Das J, Pal PB and Sil PC: Oxidative stress: The mitochondria-dependent and mitochondria-independent pathways of apoptosis. Arch Toxicol 87: 1157-1180, 2013.

27. Wang DG, Wang S, Huang B and Liu F: Roles of cellular heterogeneity, intrinsic and extrinsic noise in variability of p53 oscillation. Sci Rep 9: 5883, 2019.
28. Mi L, Xiao Z, Hood BL, Dakshanamurthy S, Wang X, Govind S, Conrads TP, Veenstra TD and Chung FL: Covalent binding to tubulin by isothiocyanates. A mechanism of cell growth arrest and apoptosis. J Biol Chem 283: 22136-22146, 2008.

(i) (3) This work is licensed under a Creative Commons Attribution-NonCommercial-NoDerivatives 4.0 International (CC BY-NC-ND 4.0) License. 